\title{
Monitoring Respiratory Syncytial Virus Regionally In Children Aged < 5 Years Old Using Emergency Department and Urgent Care Center Chief Complaint Data in Florida's Syndromic Surveillance System, Week 1, 2010 - Week 32, 2014
}

\author{
Heather Rubino*, David Atrubin and Janet J. Hamilton \\ Epidemiology, Florida Department of Health, Tallahassee, FL, USA
}

\begin{abstract}
Objective
In Florida, pre-approval of prophylactic treatment by insurance companies is tied to seasonality. Previous analyses determined that Florida's syndromic surveillance system (Electronic Surveillance System for the Early Notification of Community-based Epidemics [ESSENCE-FL]) was capable of monitoring Florida's statewide RSV seasonality. This analysis aims to determine if ESSENCE-FL can also be used to describe RSV and RSV-associated hospitalizations in children $<5$ years by region and season.
\end{abstract}

\section{Introduction}

National studies estimate that respiratory syncytial virus (RSV) is responsible for one in 38 emergency department (ED) visits for children $<5$ years old. The Council for State and Territorial Epidemiologists position statement (13-ID-07): "RSV-Associated Pediatric Mortality" advocates for improved RSV surveillance including monitoring of RSV-associated pediatric mortality and hospitalizations. The goal of that data collection is to establish prevaccine baselines to evaluate vaccine effectiveness should one become available. As RSV is not reportable in Florida, RSV surveillance relies on a small subset of all Florida hospital laboratories to report data in aggregate and calculation of percent positive of all tests for RSV performed. These data assess virus activity, and do not allow for assessment of morbidity or agespecific analysis. Moreover, this data is not complete or timely, most often becoming available a minimum of a week after the testing was conducted. Florida's RSV surveillance efforts guide clinical decision making and insurance reimbursements. Florida's RSV seasonality not only differs from the nation but there is strong variation among five distinct regions, as exemplified by southeast Florida where the RSV season is year round. In Florida, pre-approval of prophylactic treatment by insurance companies is tied to seasonality.

\section{Methods}

ESSENCE-FL was used to identify the number of visits to EDs and urgent care centers (UCCs) with RSV listed in the chief complaint or discharge diagnosis between Week 1, 2010 and Week 32, 2014. From a subset of the participating hospitals, ESSENCE-FL also provided information on admission status for children $<5$ years old. Chief complaint data were available for all 213 facilities participating in ESSENCE-FL (180 EDs and 30 UCCs), admissions data were available for 171 participating facilities, and discharge diagnosis was available for some facilities. All of these data elements were sorted by RSV region.

\section{Results}

A total of 28,779 visits to EDs and UCCs were identified with a discharge diagnosis of RSV; children $<5$ years old accounted for $27,153(94 \%)$ of all visits and children $<1$ year old accounted for $17,574(61 \%)$ of all visits. Ten percent of ED and UCC visits of children $<5$ years old with a discharge diagnosis of RSV resulted in hospital admission. Children $<1$ year old accounted for $77 \%$ of those admissions. Trend analysis showed that chief complaint and discharge diagnosis data in children $<5$ showed a high correlation with laboratory surveillance data from the state's hospital-based RSV surveillance program. For each region, ESSENCE-FL data mirrored the existing knowledge about unique seasonality.

\section{Conclusions}

ED chief complaint and discharge diagnosis data accessed through a syndromic surveillance system can be used for effective, timely monitoring of RSV hospitalizations in children $<5$ years old and may be a more efficient and complete means of monitoring seasonality of RSV activity by region and statewide compared to hospital-based laboratory data reporting. Additionally, this surveillance technique can efficiently monitor RSV activity as well as estimate hospital admissions due to RSV and may be a useful approach for other states with syndromic surveillance systems.

\section{Keywords}

Respiratory Syncytial Virus; syndromic; RSV; surveillance; seasonality

\section{*Heather Rubino}

E-mail: heather.rubino@flhealth.gov 